\title{
Nucleosynthesis in the Neutrino Driven Winds of Protoneutron Stars
}

\author{
Luke Roberts** \\ Dept.of Astronomy \& Astrophysics, University of California Santa Cruz, Santa Cruz, CA 95064 \\ USA \\ E-mail: lroberts@ucolick.org
}

\section{Stan Woosley}

Dept.of Astronomy \& Astrophysics, University of California Santa Cruz, Santa Cruz, CA, 95064

USA

\section{Alexander Heger}

School of Physics \& Astronomy, University of Minnesota, Minneapolis, MN 55455 USA

\section{Rob Hoffman}

N Division, L-414, Lawrence Livermore National Laboratory, Livermore, CA 94550 USA

\begin{abstract}
Using the stellar evolution code Kepler, which includes a full adaptive nuclear reaction network, we have performed calculations of neutrino driven winds from the surface of protoneutron stars which include hydrodynamics, all strong processes, and all weak processes of importance. In this environment, neutrino interactions push the material in the wind to high entropies and at later times weak charged current interactions tend to reduce the electron fraction to values possibly favorable for an r-process. We have also included time dependent neutrino luminosities and energies so as to follow the evolution of the wind from the proton rich stage through the neutron rich stage to calculate integrated nucleosynthetic yields. Here, we report preliminary results of our calculations and discuss future directions for this work.
\end{abstract}

10th Symposium on Nuclei in the Cosmos

July 27 - August 12008

Mackinac Island, Michigan, USA

\footnotetext{
${ }^{*}$ Speaker.

${ }^{\dagger}$ This work has been done with support from the NNSA Stewardship Science Graduate Fellowship and the DOE SciDAC program grant number DE-FC02-06ER41438.
} 


\begin{tabular}{ccccccc}
\hline $\begin{array}{c}t_{\text {bounce }} \\
(\mathrm{s})\end{array}$ & $\begin{array}{c}L_{v_{e}} \\
\left(10^{51} \mathrm{ergs} \mathrm{s}^{-1}\right)\end{array}$ & $\begin{array}{c}T_{v_{e}} \\
(\mathrm{MeV})\end{array}$ & $\begin{array}{c}L_{\bar{v}_{e}} \\
\left(10^{51} \mathrm{ergs} \mathrm{s}^{-1}\right)\end{array}$ & $\begin{array}{c}T_{\bar{v}_{e}} \\
(\mathrm{MeV})\end{array}$ & $\begin{array}{c}L_{v_{\mu}} \\
\left(10^{51} \mathrm{ergs} \mathrm{s}^{-1}\right)\end{array}$ & $\begin{array}{c}T_{v_{\mu}} \\
(\mathrm{MeV})\end{array}$ \\
\hline 0.2 & 43.2 & 3.7 & 40.0 & 4.0 & 58.8 & 6.3 \\
1.1 & 6.6 & 4.8 & 7.6 & 6.3 & 8.6 & 8.7 \\
2.7 & 2.3 & 4.2 & 2.8 & 6.3 & 3.5 & 9.9 \\
3.8 & 1.5 & 4.1 & 1.9 & 6.6 & 2.2 & 10.6 \\
\hline
\end{tabular}

Table 1: Imposed neutrino temperatures and luminosities at various time in the simulation, taken from [7]. The neutrino spectra are assumed to follow effective fermi-dirac distributions with a degeneracy parameter of zero. All $\mu$ and $\tau$ neutrino luminosities and temperatures are assumed equal.

\section{Introduction}

After a successful shock is launched in a core collapse supernova, it is expected that for a certain progenitor mass range a proto-neutron star is left inside a newly formed low-density environment behind the shock. There, the proto-neutron star begins cooling via the emission of neutrinos of all flavors. This gravitational contraction releases $\sim 10^{53} \mathrm{ergs}$ in neutrinos over $\sim 20$ seconds. These neutrinos deposit energy in the envelope of material near the surface of the neutron star via charged and neutral current interactions and drive a baryonic wind.

Early on during this wind, while the de-leptonization of the proto-neutron star is occurring, the material is driven proton rich by weak charged current interactions in the wind and conditions become favorable for the production of light p-nuclides. After de-leptonization has occurred, the wind becomes neutron rich due to a temperature asymmetry between electron neutrinos and antineutrinos. At this point conditions become favorable for an r-process to occur.

Although this is an appealing site, to date, calculations of the neutrino driven wind have not been able to reproduce the conditions required for the r-process ( see e.g. [1][2]). These calculations have not followed the nuclear evolution of the material. Rather, they have relied on seperate, parameterized studies of the r-process [3] to determine if sufficient conditions were achieved. Other works have shown that light p-elements can be produced during the early phases of the wind [4][5].

\section{Methods}

The code used for this study, Kepler, is a fully implicit lagrangian spherically symmetric hydrodynamics code that has been used in the past for stellar structure calculations and modeling of type I X-ray bursts [8][9]. To start the neutrino driven wind problem, an atmosphere of 0.01 solar masses is allowed to relax to hydrostatic equilibrium on top of an inner boundary condition simulating a 1.4 solar mass neutron star with a radius of $10 \mathrm{~km}$. Once hydrostatic equilibrium is achieved, a neutrino flux is turned on that deposits energy in the atmosphere and creates a pressure driven wind. We let this wind relax to a quasi-steady state, and then nuclear reactions are turned on. The wind is once again allowed to reach a quasi-steady state. At this point, the neutrino flux is allowed to vary with time and we calculate the nuclear burning that occurs in the wind.

Nucleosynthesis in the wind is calculated using an adaptive nuclear reaction network that can include an arbitrary number of isotopes. A smaller network is used to follow nuclear energy generation in the wind. Where available, experimentally measured nuclear reaction rates are used. When 


\begin{tabular}{cccccc}
\hline $\begin{array}{c}t_{\text {bounce }} \\
(\mathrm{s})\end{array}$ & $\begin{array}{c}T_{\text {base }} \\
\left(10^{9} \mathrm{~K}\right)\end{array}$ & $\begin{array}{c}\dot{M} \\
\left(M_{\odot} \mathrm{s}^{-1}\right)\end{array}$ & $\begin{array}{c}\tau_{\text {dyn }} \\
(\mathrm{ms})\end{array}$ & $\begin{array}{c}S_{\text {asymptotic }} \\
k_{B} \text { baryon }^{-1}\end{array}$ & $Y_{e}$ \\
\hline 0.2 & 72.8 & $2.64 \times 10^{-3}$ & 5.1 & 67 & 0.574 \\
1.1 & 54.7 & $3.14 \times 10^{-4}$ & 8.1 & 80 & 0.468 \\
2.7 & 46.3 & $4.88 \times 10^{-5}$ & 18.9 & 92 & 0.453 \\
3.8 & 43.3 & $2.56 \times 10^{-5}$ & 26.9 & 96 & 0.439 \\
\hline
\end{tabular}

Table 2: Wind conditions at various times post-bounce. Here, the dynamical timescale is defined as $\tau_{d y n}=$ $\left.\frac{r}{v}\right|_{T \approx 5 G K}$.

these are unavailable, HF calculations from [6] are used. Electron neutrino and anti-neutrino capture reactions on all nuclei are also in the network. During our calculations, the network contains at most 2000 nuclei.

Electron, mu, and tau neutrinos and anti-neutrinos are included in the calculations. Energy deposition in the proto-neutron star envelope by electron neutrino and anti-neutrino captures on nucleons, neutrino annihilation, and neutrino scattering on electrons are taken into account using the expressions given in [1]. The transport of all neutrino species is treated in the vacuum approximation, which alleviates the need to include full neutrino transport in the code. This is a valid approximation for all the processes except neutrino annihilation, as the neutrino optical depth of the wind is significantly less than unity. The neutrino anti-neutrino annihilation rate strongly depends on the first and second angular moments of the neutrino distribution, and therefore the energy deposition rate from this process strongly depends on how the neutrino transport is handled [10].

For the models presented here, we use the neutrino luminosities and temperatures given in [7]. Luminosities and temperatures at various times are listed in table 1. In the future, we plan to use trajectories from other supernova and neutron star cooling models as well. Calculations will also be performed for the quasi-steady state conditions encountered at later times in the wind.

\section{Preliminary Results}

At this time, only preliminary results are available. Calculations have been run using neutrino luminosities and temperatures taken from [7] from 0.2 seconds until $\sim 4$ seconds after bounce. The thermal state of the wind at various times is given in table 2. The wind structure at 2.2 seconds is shown in figure 1. The bump in the nuclear energy generation rate at a radius of $\sim 10^{7} \mathrm{~cm}$ is due to free nucleons forming alpha particles and heavier nuclei. After this point, the neutrino cooling rate significantly decreases due to the decrease in free nucleons available for electron and positron capture. The asymptotic entropies obtained at later times are commensurate with the calculations of [1] and [2]. Given these entropies and electron fractions, r-process nucleosynthesis is not expected to occur. At earlier times, conditions are favorable for the production of the light p-nuclides.

Figure 2 shows the decayed production factors relative to solar over the whole wind at the time the calculation is stopped. The p-nuclides ${ }^{96,98} \mathrm{Ru}$ and ${ }^{92,94} \mathrm{Mo}$ have production factors of $\sim 1000$. These elements are produced during the first second when the wind is proton rich. Also note that $\mathrm{Zn}, \mathrm{Ge}, \mathrm{As}, \mathrm{Se}$ and $\mathrm{Kr}$ have similar production factors. During the period that has been run, no significant r-process abundances are produced. There is large overproduction the elements $\mathrm{Sr}, \mathrm{Y}$, and $\mathrm{Zr}$, as has been seen in past studies [3]. These production factors are significantly larger 


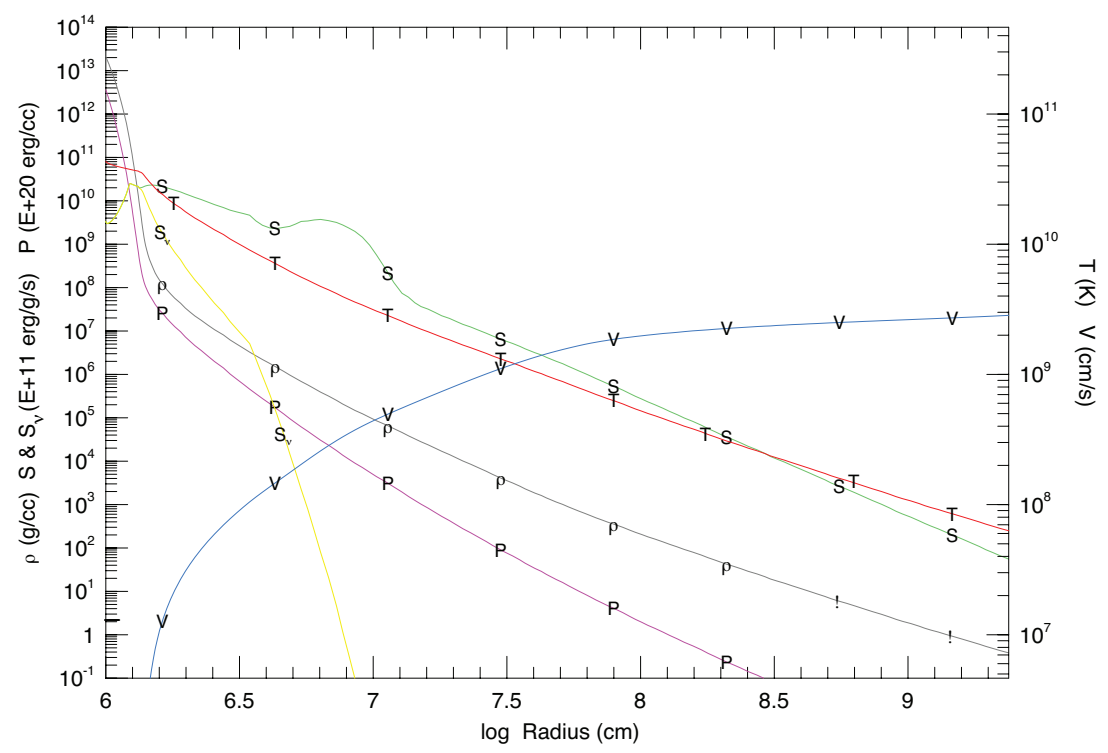

Figure 1: Thermal structure of the wind at 3.8 seconds. $S$ is the nuclear energy generation rate combined with the neutrino energy deposition and $S_{v}$ is the energy loss rate by neutrinos.

than the production factors of the light p-nuclides. During the neutron rich period, the dynamical timescale is too long and the entropy too low for an alpha-rich freeze out. Therefore, the neutron to seed ratio after charged particle reaction freeze out is too low for significant neutron addition to occur. Material then builds up in $\mathrm{Sr}, \mathrm{Y}$, and $\mathrm{Zr}$, and overwhelms the earlier production of the light p-elements.

\section{Future Directions}

Currently, we are including updated neutrino interaction physics in the code. Neutrino-nucleon capture rates will be changed so as to include corrections for nucleon recoil and weak magnetism [11] and the rates will be generalized to be able to deal with non-vacuum transport. Neutrinoelectron inelastic scattering rates will also be updated and neutrino-nucleon scattering will be included. The neutrino annihilation rates will also be changed to take into account non-vacuum neutrino transport. Once this is implemented, steady state wind configurations and nucleosynthesis for late times in the wind will be investigated, possibly coupled to the results of calculations of full neutrino transport. We also plan to run calculations with various time dependent neutrino luminosities assuming vacuum transport.

\section{References}

[1] Qian, Y.-Z., \& Woosley, S. E. 1996, ApJ, 471, 331. 


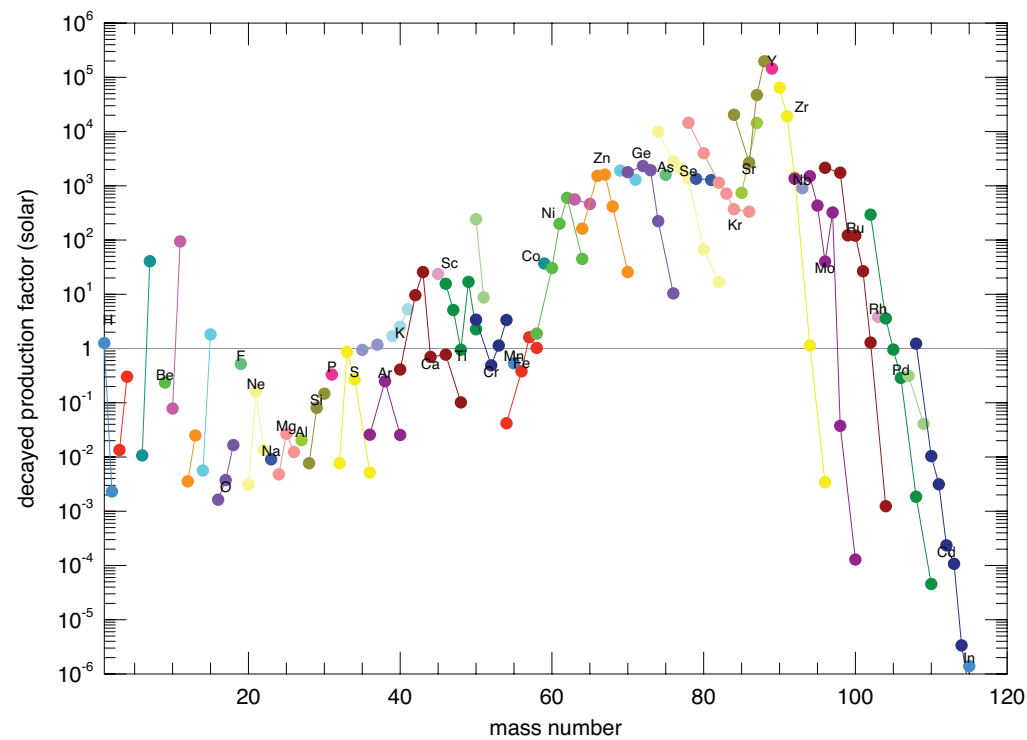

Figure 2: Isotopic production factors relative to solar of the wind composition at 3.8 seconds.

[2] Thompson, T.A., Burrows, A. \& Meyer, B.S. 2001 ApJ, 562, 887.

[3] Hoffman, R. D., Woosley, S. E., \& Qian, Y.-Z. 1997 ApJ, 482, 951.

[4] Pruet, J. et al. 2006 ApJ, 644, 1028.

[5] Froehlich, C., et al. 2006 PhRvL, 96, 142502.

[6] Rauscher, T., \& Thielemann, F. 2000 ADNDT, 75, 1-2.

[7] Woosley, S. E., et al. 1994 ApJ, 433, 229.

[8] Woosley, S. E., et al. 2004, ApJS, 151, 75

[9] Woosley, S. E., \& Weaver, T. A. 1995, ApJS, 101, 181

[10] Janka, H.-T. 1991, A\&A, 244, 378

[11] Horowitz, C. J. 1999, PhRvC, 60, 022801 